\title{
COMENTÁRIOS A RESPEITO do ARTIGO: "QUANDO EXPRESSÕES NOMINAIS Quantificadas Podem SER TOPICALIZADAS" DE ANA CAROLINA N. DE AGUIAR \& Cilene Rodrigues
}

Some comments on Aguiar \& Rodrigues's article 'Quando Expressões Nominais Quantificadas podem ser topicalizadas'

\author{
Marina Rosa Ana Augusto*
}

\section{INTRODUÇÃo}

O trabalho de Ana Carolina Nunes de Aguiar \& Cilene Rodrigues ${ }^{1}$ debruça-se sobre estruturas de tópico, enfocando, particularmente a impossibilidade, largamente tratada na literatura, de um tópico poder ser quantificado (os exemplos a seguir são retirados do artigo de Aguiar \& Rodrigues):

(1) a. *Ninguém, eu beijei.

b. * Every girl, John wants to date.

Isso se dá, conforme assume a literatura, porque expressões quantificadas são consideradas não referenciais (HEIM; KRATZER, 1998; LARSON; SEGAL, 1995) e tópicos, por sua vez, apresentam uma restrição semântica em termos de um traço de definitude/especificidade.

As autoras, no entanto, apontam para uma série de construções em que uma expressão nominal quantificada parece poder ocorrer em posição de tópico:

\footnotetext{
- UERJ.

${ }^{1}$ Trata-se de comunicação apresentada durante o Encontro do GT Teoria da Gramática de 2007.
} 
(2) Nenhum rapaz de Tiros/que o Paulo levou lá em casa, a Silvia namoraria.

Além de apresentarem estruturas em que a expressão nominal quantificada está acompanhada de um modificador, as autoras defendem que também quantificadores nus, sem modificação interna explícita, são licenciados em posição de tópico, quando houver uma retomada de contexto (ou seja, em contextos de pergunta-resposta):

(3) A: Você já assistiu os filmes do Almodóvar?

B: Vários, eu já assisti.

As autoras propõem, então, que uma estrutura larsoniana, que permite que os traços do DP sejam computados composicionalmente, dá conta de explicar a aceitabilidade de casos como os em (2) e em (3). Os modificadores são diretamente concatenados ao determinante, que se move posteriormente para a posição de núcleo do DP. Tem-se, assim, uma estrutura partitiva. O caso dos quantificadores nus (3) é visto, portanto, como um subcaso de (2), em que se obteve uma elisão de parte da estrutura nominal partitiva.

Considero que a proposta de análise via concha larsoniana para o DP é extremamente bem-vinda, ao possivelmente permitir a computação composicional dos traços dessa categoria. Abordo, no entanto, a necessidade de se precisar a natureza da restrição semântica imposta aos tópicos, embora a literatura frequentemente utilize os termos definido e/ou específico indistintamente. Verifico, então, em que medida essa precisão pode comprometer ou exigir adaptações à estrutura de DP larsoniana adotada. Apresento, ainda, neste artigo, algumas considerações que vão em sentido contrário à ideia de atribuir às construções em (3) o mesmo estatuto das estruturas em (2), com base no resultado da aplicação de alguns testes (KATO, 1998), em que características típicas das construções de tópico são checadas.

A próxima seção traz, justamente, os resultados da aplicação desses testes. A seção seguinte trata da distinção definido/específico e retoma a estrutura do DP com modificadores à la Larson. A retomada dos principais pontos e uma breve conclusão das reflexões aqui sugeridas finalizam o artigo.

\section{AplicAndo Testes de Topicidade Às EXPREssões Sob AnÁlise}

Retomamos aqui as expressões nominais consideradas e submetemô-las a alguns dos testes típicos de estruturas de tópicos.

Seguindo Kato (1998), pode-se verificar que algumas características atribuídas às construções de tópico podem ser estendidas aos DPs quantificados com modificadores explícitos: 
a) retomada por pronomes resumptivos:

(4) a. Os meninos, os professores os ouviram atentamente.

b. $\quad$ *Poucos meninos, os professores os ouviram atentamente.

c. ?Poucos/Alguns/Vários/Muitos meninos da Turma 6, os professores os ouviram atentamente.

b) Efeitos de weak crossover

(5) a. O menino, a mãe dele o admira.

b. $\quad$ Nenhum menino, a mãe dele o admira.

c. Nenhum menino que eu encontrei na Febem, a mãe dele o admira.

c) Sem efeito de ilha

(6) a. O menino, eu conheço um professor que o elogiou.

b. *Nenhum menino, eu conheço um professor que o elogiou.

c. Nenhum menino da Turma 6, eu conheço um professor que o elogiou/tenha elogiado.

No entanto, quando aplicados a quantificadores nus, os testes não parecem resultar em sentenças aceitáveis. Mantemos, como sugerido pelas autoras, o contexto de pergunta-resposta para esse caso:

a) retomada por pronomes resumptivos:

(7) A: Os professores assistiram os filmes do

Almodóvar?

B: $\quad$ *Poucos, os professores os assistiram.

b) Efeitos de weak crossover

(8) A: As mães admiram os filhos da era moderna?

B: $\quad$ *Nenhum, as mães deles os admiram.

c) Sem efeito de ilha

(9) A: Você conhece o professor que elogiou os meninos da Turma 6?

B: $\quad$ *Nenhum/Vários, eu conheço o professor que $\mathrm{o}(\mathrm{s})$ elogiou.

Em suma, esses testes não parecem corroborar a hipótese das autoras de que a presença de quantificadores nus em contexto perguntaresposta possa ser tomada como um subcaso da generalização estabelecida de que expressões nominais quantificadas, quando acompanhadas por um modificador, podem desempenhar a função de tópico. As próprias autoras admitem (em nota, no texto original) que esses casos poderiam constituir estruturas com movimento para a posição de foco, mas descartam essa possibilidade em busca de determinar que estaríamos frente a dois subcasos de um mesmo fenômeno. 
Chama a atenção, no entanto, que os quantificadores nus só sejam licenciados em contextos de pergunta-resposta, o que pode ser tomado como um forte indício de que se trata realmente de foco e não tópico. Retomemos alguns dos exemplos:

$\begin{array}{lll}\text { (10) A: } & \text { Você já assistiu os filmes do Almodóvar? } \\ \text { B: } & \text { Vários, eu já assisti } \\ \text { (11) A: } & \text { Você viu os alunos de linguística na palestra do } \\ & \text { Damasceno? }\end{array}$

Se adotarmos um contexto de interrogativa-QU, não parece haver outra possibilidade além de considerar que o quantificador nu seja o foco da sentença:

$$
\begin{aligned}
& \text { A: Que filmes do Almodóvar você já assistiu? } \\
& \text { B: } \quad \text { Vários, eu já assisti./Eu já assisti vários. }
\end{aligned}
$$

As noções de tópico e foco constituem conceitos discursivos. No que concerne ao foco especificamente, assume-se que o foco é a parte da sentença que introduz informação nova em oposição à informação que já é compartilhada pelos interlocutores - a pressuposição. De modo geral, dois tipos de focos são propostos na literatura: foco de informação e foco contrastivo. $O$ primeiro se limita a fornecer informação nova, tipicamente o que resulta no contexto de uma interrogativa-QU, como visto acima em $(12)^{2}$. O foco contrastivo, por sua vez, corrige uma informação anteriormente apresentada no discurso, caracterizando-se, geralmente, por um contorno prosódico mais acentuado. Zubizarreta (1998) distingue os dois tipos de foco por meio da noção de que o foco contrastivo contém a negação de um valor já atribuído e oferece um novo valor a ser assumido:

(13) A: O Pedro comprou um carro.

B: $\quad$ Não, o Pedro comprou [F UMA MOTO] (e não um carro).

Kiss (1998), no entanto, propõe uma distinção entre foco de informação e foco de identificação com base na noção de exaustividade. $O$ foco de identificação expressa exaustividade ao identificar um referente único (um subconjunto exaustivo) no contexto a ser interpretado como foco, devendo ser lido, portanto, como $[x$ e apenas $x]$ :

(14) A: o Pedro comprou um carro e uma moto. B: $\quad$ Não, foi uma moto que o Pedro comprou.

${ }^{2}$ Semelhante ao foco de informação, há de se fazer referência ao foco apresentativo. Conforme esclarece Mioto (2003), o foco apresentativo introduz um novo indivíduo no universo do discurso, normalmente através de um DP indefinido:

(i) $\quad$ Era uma vez ${ }_{\mathrm{F}}$ um rei] 
Com base em situações pragmáticas distintas e uma vez que se possa conciliar as posições de Zubizarreta (1998) e Kiss (1998), Mioto (2003, p. 175) compõe o seguinte quadro com os tipos de foco:

\begin{tabular}{|l|l|}
\hline a. [-contrastivo, - exaustivo] & informação (K), não-contrastivo (Z) \\
\hline b. [-contrastivo, + exaustivo] & de identificação (K) \\
\hline c. [+contrastivo, - exaustivo] & ${ }^{*}$ \\
\hline d. [+ contrastivo, + exaustivo] & contrastivo (Z) e (K) \\
\hline
\end{tabular}

FIGURA 1 - TIPOS DE FOCO (MIOTO, 2003, p. 175).

No contexto de perguntas sim/não sugerido pelas autoras (cf. exemplos (10) e (11)), uma leitura de foco de identificação, isto é, exaustivo parece ser bastante apropriada. Nessas sentenças, o quantificador em foco tem o caráter de exaurir, restringir um conjunto previamente sugerido, excluindo-se a possibilidade de se inferir desta proposição senão a totalidade dos elementos deste conjunto: não são todos os filmes de Almodóvar ou todos os alunos de linguística que foram vistos. É necessário precisar essa informação por meio do quantificador, trata-se de uma informação nova que é exaustiva em relação ao assunto filmes de Almodóvar ou alunos de linguística. Sendo assim, considero que, embora possamos tomar as sentenças com quantificadores e modificadores explícitos como casos legítimos de topicalização, conforme defendem as autoras, o mesmo não se estende aos casos de quantificadores nus em contexto de pergunta-resposta.

\section{O TRAÇO [+ESPECífICO] ASSOCIADo A tóPICOS}

Uma questão que o trabalho de Aguiar \& Rodrigues suscita é a definição ou a necessidade de busca de maior precisão no que diz respeito ao conjunto de traços que podemos atribuir aos elementos do DP. Os traçosj relativos aos nominais têm recebido atenção no minimalismo desde o texto clássico de Chomsky (1995) e, no português, tem sido tema de algumas propostas (LOPES, 2004; MAGALHÃES, 2004; AUGUSTO, FERRARI-NETO; CORRÊA, 2006; dentre outros). No entanto, não se definiu, ainda, que traços devem ser especificamente associados a $\mathrm{D}$. Se levarmos em conta a sugestão lançada no arcabouço minimalista de que toda projeção funcional deve apresentar um traço semanticamente interpretável que a justifique, então podemos nos questionar que traço justifica a projeção de D: referencialidade, definitude, indefinitude, especificidade?

$\mathrm{Na}$ literatura sobre tópico, uma indistinção entre os termos definido e específico é comumente encontrada. As autoras também mencionam a necessidade de que o "tópico possua um traço [+ definido]", remetendo às seguintes sentenças:

(15) a. Estas crianças, a professora certamente aprovará.

b. These kids, the teacher will certainly approve. 
No entanto, faz-se necessário que uma distinção mais acurada entre definitude e especificidade seja estabelecida: nem todo DP definido é necessariamente específico. Ihsane \& Puskás (2001) fornecem um exemplo, adaptável ao português:

(16) Perdi o trem (do metrô).

Embora estejamos diante de um DP definido, podemos ter uma leitura não específica. Cheguei e havia um trem partindo que eu não consegui tomar - não se trata de um trem específico, mas qualquer trem que poderia ter passado naquele momento.

Esse tipo de leitura, no entanto, é restringida pela posição sintática. As autoras observam, em relação ao húngaro, que, ao ser topicalizado, um DP definido como esse só pode ser interpretado como específico. Enquanto (16) é ambígua, (17) só admite a leitura específica:

O trem, eu acabei de perder.

Logo, tanto DPs definidos quanto DPs indefinidos podem, a priori, ser específicos ou não específicos. No que diz respeito a tópicos, a restrição parece ser em relação à especificidade (cf. AUGUSTO, 2003; 2009). Augusto (2003, 2009) assume que verbos factivos, que pressupõem a verdade de seus complementos, apresentam uma restrição selecional em termos de [especificidade]. Sendo assim, verifica-se que um DP indefinido não é aceito como complemento de um verbo factivo. No entanto, se modificado por uma relativa, esse complemento satisfaz as imposições de um predicado factivo:

(18) a. *Pedro lamentou uma briga.

b. Pedro lamentou uma briga que ele teve com o seu chefe.

Segundo Fodor \& Sag (1982), "any relative clause modifying an indefinite adds to its descriptive content and thus tends to favor a referential understanding".

Em suma, a presença de DPs indefinidos, porém específicos, é possível na posição de tópico:

(19) Uma criança, a professora certamente aprovará.

Essa sentença é plenamente aceitável em um contexto em que uma diretora apresenta uma classe com crianças com rendimento muito fraco à inspetora, comentando que não acredita que nenhuma criança será aprovada pela nova professora. A inspetora, no entanto, reconhece entre esses alunos uma criança que, ela sabe, é boa aluna. Nesse contexto, a sentença apresentando um DP com artigo indefinido (ou numeral, nesse caso) tem 
uma interpretação específica. Não se pode atribuir à "uma criança" o traço definido, mas com certeza a leitura possível aqui é de que há uma criança específica que será aprovada.

Sendo assim, a noção mais adequada a adotar é a da especificidade, como o traço relevante que possibilita que DPs indefinidos ou quantificados apareçam na posição de tópico. Inclusive, a noção de especificidade é relevante em relação à estrutura de partitividade que, segundo as autoras, seria a estrutura de DPs quantificados com modificador explícito:

\section{(20) Rasguei uma página do meu livro.}

A estrutura assumida pelas autoras a partir de Larson $(1991,2005)$ assume uma concha com duas camadas relacionadas ao DP, sendo que a camada mais alta é denominada äP pelas autoras. A proposta de Larson (1991, 2005) é derivada da assunção de que os determinantes também projetam uma estrutura argumental, o que os aproxima configuracionalmente da estrutura proposta para o domínio verbal, ou seja, assim como VPs, DPs são conchas larsonianas. O determinante é gerado na camada mais baixa, movendo-se depois para a camada mais alta. Em relação aos DPs com modificadores, Aguiar \& Rodrigues sugerem, seguindo Larson, que os argumentos internos ao DP sejam tematicamente marcados com os papéis temáticos de restritor (o sintagma nominal) e oblíquo (o sintagma preposicional), conforme representação a seguir:

(21)

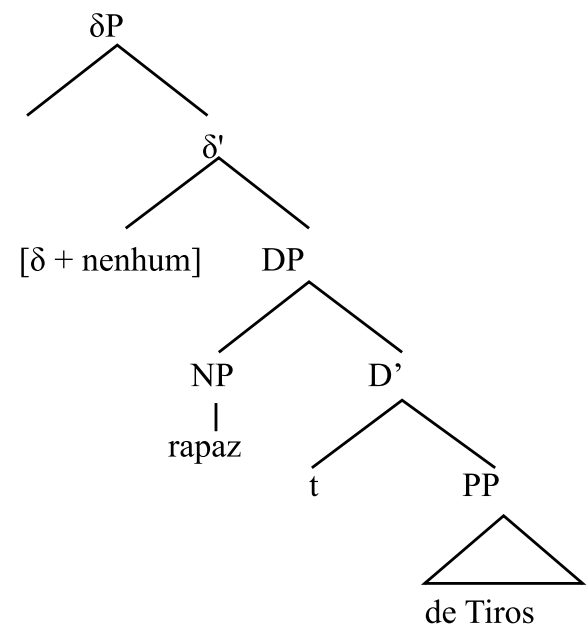

Essa estrutura também se mostra interessante em relação aos DPs modificados por relativas. Conforme mencionado, a relativa parece dotar um DP indefinido do traço de [+ específico] (cf. (18)). Recentemente, tem-se adotado, para a estrutura relativa, uma configuração em que a sentença relativa é complemento de D, conforme sugestão de Kayne (1994). 
(22)

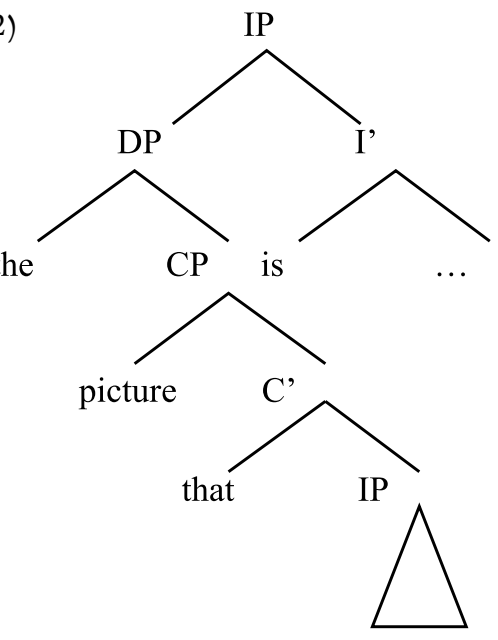

Bill saw [NP picture]

Horsntein (2001), no entanto, prefere manter uma estrutura de adjunção para as relativas, apresentando algumas objeções em relação à estrutura kayniana, que merecem atenção. Para Hornstein (2001), a derivação de uma relativa como:

(23) John met every man who Bill likes t

envolve, após movimento do argumento do verbo da relativa para $\mathrm{CP}$, que este seja copiado e adjungido ao CP. Em seguida, every é retirado da Numeração e concatenado à estrutura formada até então:

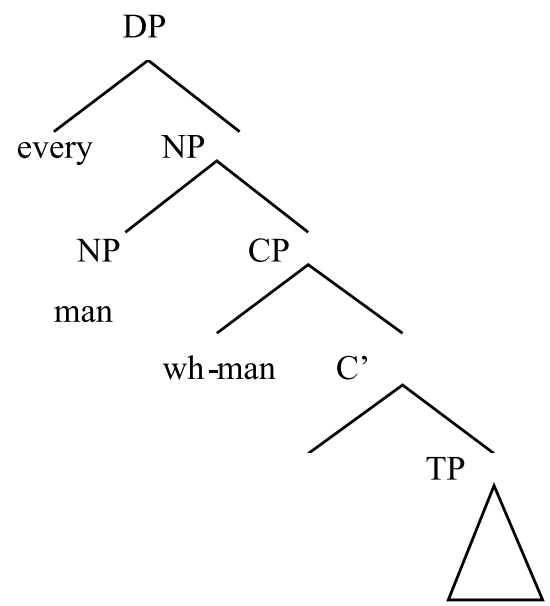

Bill likes wh-man 
Essa estrutura mantém a ideia de que a relativa forma uma estrutura de adjunção (NP/NP), embora mantenha o núcleo nominal e a relativa como um constituinte. Segundo Hornstein (2001), as evidências que favorecem essa análise dizem respeito ao fato de que relativas podem ser retomadas por anáforas do tipo one. Esse tipo de elemento, no entanto, retoma sempre NPS (HORNSTEIN; LIGHTFOOT, 1981), mas não CPs ou DPs (exemplo (70) no original):

(25) John saw this man who Frank photographed and Bill saw that one.

Outra evidência consiste na possibilidade da relativa aparecer mais à direita na estrutura, separada do núcleo, ou seja, também o determinante e o núcleo devem formar um constituinte:

(26) I gave several spiders to Max that he loved.

$\mathrm{Na}$ estrutura kayniana, one estaria retomando um $\mathrm{CP}$ e that he loved não formaria um constituinte independente, que possibilitasse a extraposição.

No entanto, no que diz respeito à estrutura do DP larsoniana, parece que uma aproximação com a estrutura kayniana poderia ser alcançada em relação às relativas. Para tanto, assumir-se-ia o movimento do nome interno ao $\mathrm{CP}$ para a posição de restritor na concha larsoniana e posterior movimento do determinante para a camada mais alta do DP. Essa estrutura estaria isenta das objeções apresentadas por Hornstein, mantendo-se, no entanto, a ideia de que a relativa é um complemento de $\mathrm{D}$.

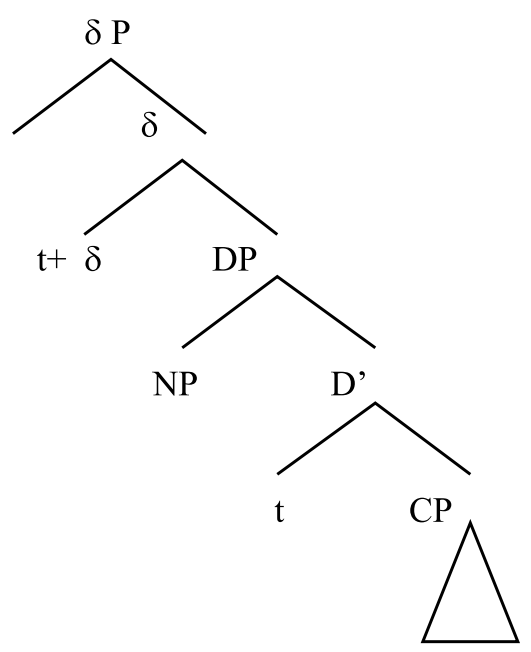

that he liked 
Parece interessante poder derivar tanto nominais com modificadores PPs, como aqueles com relativas a partir da mesma estrutura, pois conforme visto aqui ambas combinações podem levar um DP indefinido a ser interpretado como [+ específico].

Nesse sentido, as autoras sugerem que a configuração sintática proposta por Larson para a derivação desses DPs poderia permitir um cálculo de composicionalidade dos traços dessa categoria em paralelo com o que se observa no domínio verbal em relação ao aspecto verbal, em que é necessário levar em conta a presença (e o tipo) de complementos e adjuntos com os quais esses se combinam (exemplos fornecidos pelas autoras):

(28) a. O João empurrou o carro.

b. $\quad$ O João empurrou o carro até o posto de gasolina da $Q 19$.

a. O João empurrou o carro por horas/*em 40 minutos.

b. O João empurrou o carro até o posto de gasolina da Q19 \#por horas/em 40 minutos.

As autoras não definem, no entanto, que tipo de abordagem preferem adotar: sintática ou lexicalista. A opção pela primeira precisaria garantir que a proposta de Larson é uma opção teórica que permitiria tratar de questões como a estrutura argumental para o cálculo composicional do tipo de DP, ou seja, permitindo um tratamento da coerção do tipo semântico. A alternativa lexicalista, por sua vez, teria que assumir que a presença do traço [+ específico], possivelmente associado ao caráter de tópico do DP quantificado, forçaria certo tipo de derivação sintática, via estrutura sintática complexa.

Em relação a essa segunda alternativa, seria ainda necessário verificar de que maneira a estrutura nominal satisfaria a imposição lexical. Nada garante que o valor positivo do traço [específico] seja derivado da estrutura larsoniana em si. Embora haja um movimento do determinante da camada mais baixa para a camada mais alta da concha, este nada parece ter a ver com a questão da especificidade, uma vez que se faz independentemente necessário para que se obtenha a ordem canônica entre determinante e nome no $\mathrm{DP}^{3}$. Seria então necessário associar especificidade a uma camada funcional ainda mais alta? Ihsane \& Puskas (2001) adotam essa possibilidade, sugerindo a presença de uma camada mais alta no DP, associada ao traço de [especificidade], a camada TopP.

\section{CONSIDERAÇõES FinAIS}

O trabalho de Aguiar \& Rodrigues traz uma relevante contribuição para o fenômeno da topicidade ao debruçar-se sobre estruturas de tópico com quantificadores e sugerir que esses determinantes indefinidos tornam-

\footnotetext{
${ }^{3}$ Em relação à camada verbal, esse movimento pode ser motivado pelo traço de agentividade ou causalidade associado ao verbo leve - v.
} 
se definidos ao tomarem um modificador restritivo como argumento. Para tanto, as autoras exploram a estrutura de concha larsoniana para os DPs e defendem que são tópicos tanto DPs que apresentam um modificador restritivo explícito, quanto aqueles em que se tem um modificador implícito.

Nestes comentários, reiterei a possibilidade de DPs partitivos com modificadores explícitos poderem funcionar como tópicos, mas questionei essa possibilidade em relação aos quantificadores nus em contexto de pergunta-resposta, que, sugeri, constituiriam um tipo de foco de identificação. Salientei a necessidade de se precisar a natureza da restrição semântica imposta aos tópicos, argumentando a favor da noção de especificidade. Segundo as autoras, a estrutura larsoniana possibilita explorar potenciais composições de traços dos DPs. Essa possibilidade precisa, ainda, ser trabalhada a fim de se definir se realmente se poderia proceder a um cálculo composicional dos traços do DP nesse tipo de estrutura ou em que medida uma camada funcional adicional, relativa ao traço de especificidade, se faria necessária.

\title{
RESUMO
}

Este trabalho apresenta comentários a respeito do artigo "Quando Expressões Nominais Quantificadas podem ser topicalizadas" de Aguiar \& Rodrigues. As autoras exploram a estrutura de concha larsoniana para os DPs e defendem que são tópicos tanto DPs quantificados que apresentam um modificador restritivo explícito, quanto aqueles em que se tem um modificador implícito. Com base no resultado da aplicação de testes de topicidade (KATO, 1998), reitero a possibilidade de DPs partitivos com modificadores explícitos poderem funcionar como tópicos, mas questiono essa possibilidade em relação aos quantificadores nus, que, sugiro, constituem um tipo de foco de identificação (KISS, 1998). Saliento a necessidade de se precisar a natureza da restrição semântica imposta aos tópicos, argumentando a favor da noção de especificidade. Reitero a importância de as autoras definirem o tipo de análise a assumir: se lexicalista ou sintática, que implicará explicitar como a estrutura larsoniana poderia permitir um cálculo composicional dos traços do DP ou em que medida seria necessário adotar reformulações ou expansões à estrutura larsoniana.

Palavras-chave: quantificadores; tópicos; especificidade.

\begin{abstract}
Some comments on Aguiar \& Rodrigues's paper are offered. Aguiar \& Rodrigues argue that quantified DPs with an explicit
\end{abstract}


modifier as well as bare quantifiers with implicit modifiers may be topics. They assume a larsonian DP structure in which $\mathrm{D}$ takes the modifier as its complement and the noun as the restrictor. Based on some tests on topicality (KATO, 1998), I show that partitive DPs with explicit modifiers may be topics but not bare quantifiers with implicit modifiers, which I suggest are an instance of identificational focus (KISS, 1998). I argue for the need of assuming specificity as the accurate notion concerning the kind of semantic restriction imposed on topics. Based on that, I suggest that Aguiar \& Rodrigues should define how the specific restriction on topics would be achieved for partitive DPs with quantifiers from a compositional calculus on the larsonian structure or whether additional assumptions would have to be made in relation to the larsonian configuration.

Keywords: quantifiers; topics; specificity.

\section{REFERÊNCIAS}

AUGUSTO, Marina R. A. Padrões de Extração em Estruturas Factivas. Tese (Doutorado) Universidade Estadual de Campinas, 2003.

. Patterns of extraction out of factive islands in Brazilian Portuguese. In: Jairo Nunes (Org.) Minimalist Essays on Brazilian Portuguese Syntax. Holanda, John Benjamins, p. 69-92, 2009.

AUGUSTO, Marina R. A.; FERRARI-NETO, José; CORRÊA, Letícia S. Explorando o DP: a presença da categoria NumP. Revista de Estudos da Linguagem, v. 14, p. 245-276, 2006.

CHOMSKY, Noam. The minimalist program. Cambridge, Massachusetts: MIT Press, 1995.

FODOR, Janet Dean; SAG, Ivan. Referential and quantificational indefinites. Linguistics and Philosophy, n. 5, p. 355-398, 1982.

HEIM, Irene; KRATZER, Angelika. Semantics in generative grammar. Malden \& Oxford: Backwell, 1998.

HORNSTEIN, Norbert. Move! A minimalist theory of construal. Oxford: Blackwell, 2001.

HORNSTEIN, Norbert; LIGHTFOOT, David. Explanation in linguistics: The logical problem of language acquisition. London: Longman, 1981.

IHSANE, Tabea; PUSKÁS, Genoveva. Specific is not definite. Generative Grammar in Geneva, n. 2, p. 39-54, 2001.

KAYNE, Richard. The Antisymmetry of Syntax. Cambridge: The MIT Press, 1994.

KATO, Mary. Tópico como Alçamento de Predicados Secundários. Cadernos de Estudos Lingüísticos, n. 34, p. 67-76, 1998.

KISS, Katalin. Identificacional focus versus information focus. Language, v. 74, n. 2, p. 245-273, 1998.

LARSON, Richard. The Projection of DP (and DegP). Ms, SUNY - Stony Brook, 1991. 
. The Projection of the DP Structure. Lecture notes, LSA/2005, 2005.

LARSON, Richard; SEGAL, Gabriel. Knowledge and meaning. An introduction to Semantic Theory. Cambridge: The MIT Press, 1995.

LOPES, Ruth. Estágios no processo de aquisição de número no DP do português brasileiro. Letras de Hoje, Porto Alegre, v. 39, n. 3, p. 157-171, 2004.

MAGALHÃES, Telma. A valoração de traços de concordância dentro do DP. D.E.L.T.A., v. 20, n. 1, p. 149-170, 2004.

MIOTO, Carlos. Focalização e Quantificação. Revista Letras, Curitiba, n. 61, especial, p. 169-189, 2003.

ZUBIZARRETA, Maria Luisa. Prosody, Focus and Word Order. Cambridge, MA: MIT Press, 1998.

Submetido em: 08/03/2008.

Aceito em: 14/07/2009. 